\title{
Design of Enhanced RTP-RTCP Protocols for Heterogeneous Wireless Ad-hoc Networks to Enhance Channel Coverage and Capacity
}

\author{
Mohan Babu.C ${ }^{1}$, Dr.B.Sivakumar ${ }^{2}$, Pradeep Kumar $S^{3}$ Sridhar C S $^{4}$ \\ ${ }^{1}$ Research Scholar, VTU, RC-Dr.AIT, Bangalore, Belgavi, mohanbabu015@gmail.com \\ ${ }^{2}$ Professor, Dr. Ambedkar Institute of Technology, Bangalore, sivab881@gmail.com \\ ${ }^{3}$ Research scholar, VTU, RC-Dr.AIT, Bangalore, Belgavi, pradeep.appu@gmail.com \\ ${ }^{4}$ Asst.Professor, SJC Institute of Technology, Chickballapur, sridhar_cs@yahoo.com
}

\begin{abstract}
The usage of the network for cellular and Ad-HoC has becoming density due to limitations of capacity, coverage, and Quality of Service (QoS). We introduce the heterogeneous Ad-HoC wireless network for the increase of the coverage and QoS to satisfy the end-users. The proposed research work proposes Heterogeneous Real-Time Transport Protocol (HRTP) and Real-Time Transport Control Protocol (H-RTCP) which utilizes different data formats and standards. For successful packet delivery ration between sources and destinations, the combined HTRP and HRTCP are most close to IEEE 802.11 wireless mesh network. The combined routing protocol and routing algorithm are provided better Packet Delivery Ration (PDR), minimum Jitters, good end-to-end delay, and high throughput. The combined proposed heterogeneous network solves the problems of the number of packets sending over longer distances with different paths and increases the network capacity and converges. The designed heterogeneous network is validated by using the NS2 simulation environment and the obtained results are outperforming when compared with LTE and Wi-Fi technologies. The simulated results are shows that, there is an improvement of $23 \%, 45 \%, 12 \%$ and $78 \%$ in throughput, end-to-end delay, efficiency, and Jitter respectively.
\end{abstract}

Key words: Ad-HoC wireless network, Capacity of the network, Heterogeneous network, LTE, RTP, RTCP, routing protocol and Wi-Fi.

\section{INTRODUCTION}

Web traffic is relied on to manufacture three to various events all through the going with three years because of the making number of related PDAs. The measure of related gadgets and machine-to-machine exchanges is relied on to outflank the measure of the individuals by a factor of two all through the going with three years. It is anticipated that inside the following decade, a further made Internet framework will be required to help this augmentation in Internet traffic [1]. Front line remote systems need to address a few challenges, including the expense to cover high-thickness zones, swarmed occasions, enormous districts or react to passing changes searched for after e.g giant redirections. The cost estimation is subject to the amount of required base stations and the expense to lease rehash social occasions. Interoperability is another test a comparable number of contraptions utilize indisputable working frameworks, shows, and access movements. System dependable quality is in addition a basic issue that should be coordinated to guarantee structures can endure flaws if there should develop an occasion of fiascoes. In [2] presents heterogeneous structure working in which LTE and Wi-Fi remote gadgets are used to profit by the data move breaking point of the every transmission headway. Besides, another directing show for heterogeneous remote work structures is made, which picks seriously the transmission improvement to produce the general system purpose of constrainment and update the standard throughput. Furthermore, another planning calculation is proposed for the requirements of the coordinating show, which quantifies the expense of transmitting the traffic through each structure. The proposed calculation considers the traffic load on the LTE plans as an estimation to look at the expense of transmission LTE and utilizations transmission rate as estimation for the Wi-Fi work create. This work gives the establishment of future research on making heterogeneous Wi-Fi/LTE work structures and utilizing various remote degrees of progress as a section of heterogeneous systems. The proposed controlling show can be reached help various remote types of progress by using their parameters in the learning estimation. The proposed planning gives a fundamental procedure to widen the flexible structure joining and limit and could add to the $5 \mathrm{G}$ foundation. Furthermore, the heterogeneous structures could be utilized to interface the Internet of Things systems and used to give the framework to astute homes and sharp urban systems. 
Another promising remote arrangement for the top tier time of remote structures is remote work systems (WMNs). The WMN is a point of view made to give wide system thought without utilizing joined foundation [4]. Thusly, WMNs are a reachable decision to give a spine system to metropolitan area structures (MANs). In such structures, segments (remote focus focuses with a snappy wired relationship with the outside Internet) are utilized to give Internet associations with the work organize. These structure offers financially shrewd all current remote relationship with the Internet in huge territories through the multi-skip transmission to the section and the diverse way. In any case, the enormous injuries of utilizing WMNs are their hindrances to the degree to compel, framework execution, and ensured remote affiliation quality. The clarifications behind those issues start in the multi-ricochet nature of the structure. Precisely when information bundles investigate more jumps in a tremendous WMN, they may either negligence to achieve their target or devour such countless system assets. Additionally, if there should be an occasion of affiliation or focus point disappointment, two or three focus focuses may wind up detached from the system taking into account the nonappearance of a course to the goal or passage, and structure what is known as an 'island focus point'. The utilization of heterogeneous advances in remote structures improves the general system execution by dissipating the heap across over various structure improvements [5, 6, 7]. Regardless, changing start with one transmission contraption then onto the difficulties of the accompanying present as every remote gadget uses various guidelines and information social occasions. Moreover, controlling gatherings through a heterogeneous system requires another section to trade control messages among the various structures. LTE [8] and IEEE 802.11 [9] are utilized in every remote community to shape the foundation for the proposed arrangement. In like way, another coordinating show is made to draw in the correspondence among the heterogeneous remote community focuses. A lot of directing tables and control messages are utilized to deal with the interoperability of the proposed heterogeneous remote work sort out. In addition, the paper proposes another heterogeneous controlling check called Cognitive Heterogeneous Routing (CHR) that draws in heterogeneous systems to fill in as a solitary structure. The gatherings are guided through various remote headways dependent on the QoS estimations of each system. To redesign the affirmation of the transmission progression, another assistance learning estimation dependent on Q-learning is made to engage each inside point to get from past activities and improve the structure execution. Wide reenactments under various conditions and traffic request in the structures display a throughput gain with up to $200 \%$ in the proposed heterogeneous system when stood apart from LTE and Wi-Fi just structures.

There are two sorts of controlling show in WMNs. The chief sort involves responsive coordinating shows in which the course is made on enthusiasm by flooding the framework with course requests. The course assurance is kept up only for center points that transmit traffic to a particular objective.
Examples of this kind of controlling are unrehearsed on-demand expel vector (AODV) [10] and dynamic source coordinating (DSR) [11]. Responsive guiding causes some deferral in view of how a course is made exactly when there is data arranged to be sent. The second sort of guiding show involves proactive or table-driven coordinating shows. They keep up a table of the entire objective in the framework by irregularly appropriating an update of the guiding table to all center points. Objective sequenced expel vector (DSDV) [12] and improved association state controlling (OLSR) [13] are examples of this sort of coordinating show. The course table keeps up the course for each objective; transmission begins with no deferral if there are bundles arranged to be sent. Nevertheless, some overhead is incorporated for passing on coordinating table information among the center points in the framework. Some controlling shows, known as cross breed coordinating shows, join responsive and proactive coordinating to diminish the overhead obviously exposure by using proactive guiding for close center points and making courses for far centers using on enthusiasm coordinating [14].

\section{LITERATURE SURVEY}

This bit of the review discusses structures that utilization different sorts of transmission developments in remote frameworks. The heterogeneous framework models are investigated according to the kind of transmission propels used in the heterogeneous frameworks (for instance cell frameworks and $\mathrm{Wi}$-Fi devices). A cell/multi-skip Wi-Fi design is proposed to move information bundles for customers that experience the shrewd effects of low channel quality or to offload a plugged up cell by sending the traffic from this cell to other non-blocked cells [14-17]. These structures use the multi-jump Wi-Fi plan as a partner system to involve traffic starting with one cell then onto the accompanying. Heterogeneous systems split information among broadband and $\mathrm{Wi}-\mathrm{Fi}$ remote structures [5-7, 18-22] to expand the sort out purpose of constrainment. One way of thinking is to disperse traffic among systems enough [5] by utilizing load-modifying estimations. Different plans utilize remote qualities to appropriate information among systems. For example, systems with better divider entryways are used for indoor correspondence while structures with higher recurrent social occasions are utilized for outside correspondence [6,7]. Traffic need is utilized to manage bunches stream in heterogeneous structures [18] in which essentially delicate packs from the Wi-Fi system are sent through the cell system to keep up a key division from scheming affiliations. Wi-Fi segments are moreover used to make pico-cells to offload plugged up cells in cell systems [18]. In [23] showed an exceptional figuring dependent on a warm reason for area choice from a VANET structure to the LTE Advanced foundation. This new way of thinking has been wandered from the standard deterministic philosophy that utilizes the $\mathrm{CH}$ as a default door. Two groupings and $\mathrm{CH}$ affirmation calculations have been inspected and reenactment results display that our show performs better outcomes concerning deferral and bundle episode than the deterministic technique for the two figuring's. In addition, reenactments 
show that a proficient $\mathrm{CH}$ decision calculation is essential to guarantee uncommon shows relatively comparatively similarly as with C-DRIVE higher gathering misfortune midpoints have been seen than with center checks. In our future works, we will concentrate on considering the introductions of FQGWS calculation and breaking down its adaptability while performing gathering head handovers in a packaged structuring and updating the gateway bargains sort out where picked entryway competitor may be dubious for two or three source place focuses having a spot with a similar pack phenomenally if there should develop an occasion of little packs [23].

In [24] explored the plan of vitality effective cell organizes through the work of base station rest mode methodologies just as little cells and researched the tradeoff issues related to these strategies. Utilizing a stochastic geometry-based model, we determined the achievement likelihood and vitality proficiency under dozing techniques inhomogeneous macrocell and heterogeneous K-level systems. Furthermore, we planned enhancement issues as power utilization minimization and vitality proficiency amplification and decided the ideal working recurrence of the macrocell base station. Specifically, we researched the effect of irregular resting and vital dozing on power utilization and vitality productivity. Numerical outcomes affirmed the adequacy of the dozing system in homogeneous macrocell arranges however the increase in vitality productivity relies upon the kind of dozing methodology utilized. What's more, the organization of little cells by and large prompts higher vitality proficiency, however, this addition immerses as the thickness of little cell increments.

Two cell determination diversions are proposed for various system situations to portray the association practices of nonsubscribers inside the transmission scope of the femtocell base station. With the thought of plausible utility capacities for nonsubscribers, the presences of unadulterated methodology Nash equilibrium are separately demonstrated for the two cell choice amusements dependent on their particular properties. Principle numerical aftereffects of this paper can be condensed as pursues [25]:

$>$ Cross breed get to mode is better than shut access mode regardless of which viewpoint, endorsers, whole HetNet, or administrator income, is considered.

$>$ Open access mode can result in higher limit with regards to the whole HetNet and more noteworthy income for administrator contrasted with both the half and half and shut access modes.

$>$ Endorsers can acquire higher limit in the cross breed get to mode contrasted with both the open and shut access modes. In this way, it is proposed to receive cross breed get to mode so as to give higher adaptability to execution improvement all things considered, whole framework, and administrator [25].
A cross-layer plan for the competent development of sight and sound associations in heterogeneous remote structures is outlined. Composed cross-layer building fuses five layers. The proposed facilitated endeavors between the layers are principal and objective. The key thought behind the proposed cross-layer setup is to envision forefront handoffs, to provoke senders with these occasions, and to fortify them to test for the upsides of the new remote system. Trick packs are utilized along these lines. Two sorts of natural media applications are thought of, explicitly, TCP-based and RTP-based applications. For each sort, satisfactory updates are proposed. The presentation of the proposed cross-layer arrangement is reviewed for both TCP-based and RTP-based applications utilizing PC stimulations. Increase results explain the remarkable execution of the proposed cross-layer setup in accomplishing high throughputs while reducing bunch drops. The outcomes likewise show the adequacy of fabrication isolates making a cautious estimation of the accessible transmission limit. The nature of the proposed cross-layer structure to changing system conditions is comparatively avowed. The outcomes are promising for spilling sight and sound associations in heterogeneous remote structures where the uniqueness in the accessible data move limit is up to this point a significant issue to deal with [26].

Flexible adjustments of RTP and RTCP are proposed. These variations transmit sight and sound streams through an IoT space. These variations are called IoT-RTP and IoT-RTCP. The fundamental thought of these structures is the division of a colossal intuitive media session into more diminutive ones. In addition, these variations consider uncommon properties of IoT condition, for instance, coding, tolerable assortment, channels, and bottlenecks. New fields are incorporated the header of the balanced variations to choose the status of the framework at times. NS2 is used to assemble a proliferation IoT condition for testing the proposed balanced interpretations. The intentional introduction estimations are beginning to complete deferral, delay jitter, group hardship, and number of RRs, throughput, and essentialness use. The results exhibit that the proposed IoT-RTP/RTCP outmaneuvers the standard adjustments of RTP and RTCP. The rates of advancement finally to-end delay, concede jitter, number of RRs, package adversity, and throughput are $2.05 \%$, $39.14 \%, 36.61 \%, 37.42 \%$, and $16.51 \%$, separately. The rates of advancement in essentialness use for RFID, WSN, and MAN frameworks are $20.72 \%, 18.35 \%$, and $17.54 \%$, independently. The usage of IoT-RTP/RTCP to transmit intuitive media streams cautious IoT conditions is along these lines endorsed [27]. The MRTP show for progressing sight and sound vehicle over versatile extraordinarily designated frameworks is presented. Our recommendation was moved by the progressing noteworthy research effort immediately and sound exchanges with way arranged assortment. MRTP is an increase of existing steady vehicle shows (i.e., RTP/RTCP) that merges the multipath transport limit; it is moreover correlative to existing data driven vehicle shows (i.e., SCTP) for progressing blended media applications. We in like manner presented two execution examinations of the proposed show. 
The chief focus focused on the impact of traffic allocating the queuing execution of ceaseless streams at a bottleneck center point. We showed that the exchange speed utilization of a bottleneck center can be remarkably improved when MRTP is used. Similarly, the improvement can be practiced with a tolerably unassuming number of MRTP streams. The resulting examination focused on the misstep quality piece of MRTP. Through OPNET entertainments, we showed that MRTP achieves significant improvements overlooked video quality over single-way RTP [28].

To encourage the regularly developing interest for limit increments to remote associations, a trustworthy technique is to make close connections between the beneficiary and transmitter. The upgrades are twofold: higher quality connections and more prominent spatial reuse. The spatial reuse (in $\mathrm{b} / \mathrm{s} / \mathrm{Hz} / \mathrm{m} 2$ ) is promptly expressible by the territory otherworldly effectiveness (ASE) [29] which is a proportion of the all-out reachable system throughput per unit $\mathrm{Hz}$ per unit region [30]. Generally for indoor correspondence, where the interest is most noteworthy, dispersed heterogeneous femtocell frameworks (DAS), microcells, transfers, and hotspots have been proposed. Later advances in remote systems have seen the presentation of the recently made remote base station (BS) order of 'little cells' or little cell base stations (SBS) being proposed. These incorporate femtocell, Pico cells, microcells, and ultra-thick little cells where each sub-class is generally controlled by the inclusion territory or cell span it bolsters. On account of the femtocell base station (FBS), this will be in the request of many meters though Pico cell and microcell have an all-inclusive range from a couple of hundred meters to a couple of kilometers. Besides, it is generally concurred that boosting the quantity of cells with little radii will be the principle supporter of the up and coming age of cell frameworks (for example 5G) to build the system limit and to give required information rates [31]. Later most vision papers on 5G likewise bring up little cells as the key innovation to accomplish 5G necessities [31[34]. Subsequently, SBSs are relied upon to assume a fundamental job in accomplishing the $5 \mathrm{G}$ targets. Vitality effectiveness (EE) in cell systems is a developing worry for cell administrators to not just keep up benefit by limiting their operational use (OPEX) yet additionally to diminish by and large ecological impacts. The Kyoto Protocol, marked in 1997, built up lawfully restricting duties for the decrease of ozone depleting substances including carbon dioxide (CO2). With Information and Communications Technologies (ICT) delivering $2-2.5 \%$ of the complete worldwide $\mathrm{CO} 2$ emanations [35] the sum being equivalent to that of the flight business, the correspondences business has just begun making ventures towards progressively green interchanges. For instance, in the UK, Of com declared the decrease of $\mathrm{CO} 2$ discharge of cell systems to half by 2020 [36]. With the interest for progressively greener for example more vitality effective framework configuration being a key driver for ebb and flow and future remote framework configuration, arrangement of MIMO and multi-heterogeneous femtocell plots in SBSs presents a clashing worry since they accompany an expanded intricacy and vitality consumption. On account of little cells, the issue is exacerbated by the foreseen rollout.
Anticipated figures for the foreseen development of little cells have changed in the course of recent years. Ongoing figures gave [37] foresee that there will be up to 70 million little cells, including FBSs, sent by 2017 . Their interest has driven some to foresee that in the following 10-multiyear time allotment the quantity of BS including SBSs will surpass the quantity of mobile phone endorsers [38]. It is consequently certain that there is a need to address the vitality productivity of the SBS, especially outfitted with various heterogeneous femtocell, as conceivably tremendous systems of little cells are conveyed. As their organization winds up across the board, innovative difficulties will exist to keep the general carbon impression of possibly huge systems of SBSs leveled out.

Little cells are now viewed as 'green'/vitality productive [39]. This is generally identified with the way that they are near their served client gear (UEs) subsequently the required transmit power is exceptionally low when contrasted with a MBS. This is on the grounds that the way to expanding the limit is to upgrade the gathering among transmitter and beneficiary by limiting the separated and $\alpha$ the way misfortune example since way misfortune makes the sign $\operatorname{rot}$ as $\operatorname{Ad}^{\wedge}(-\alpha)$, where $A$ will be a way misfortune steady (little cells ordinarily spread an indoor scope of 50-200m for private and venture SOHO arrangements yet littler separations are foreseen with further system densification). All things considered, the primary prerequisites of a useful little cell configuration are diminished cost, size and low vitality utilization [40]. Also and a specific driver for future 5G frameworks is the low intricacy of the arrangement. Procedures that utilize numerous heterogeneous femtocell to broaden the range or increment the unearthly proficiency of the little cell, thusly, must be low in operational cost, power, and multifaceted nature.

\section{METHODOLOGY}

\subsection{The Femtocell Base Station (FBS)}

FBSs, which record for 96\% of all SBS [41], are little, economical low power base stations more often than not sent by the customer and are generally associated with the center media communications organize over the client's home wired backhaul broadband. In this regard, they look like Wi-Fi passageways [43], however rather, they use at least one business remote principles and authorized range [42]. The determination and access control to a little cell system are given by one of three access control components [44]. These are classified as - shut access or shut supporter gathering (CSG), open access or half and half access. In a CSG controlled system, just a subset of, pre-enrolled, clients can associate with a little cell though open access little cell enables all clients of the administrator to interface and utilize the little cell. In cross breed get to, a predetermined number of little cell assets are accessible to all clients though the rest of in a CSG way [43].

\subsection{Heterogeneous Networks}

To address capacity demand, SBSs including FBSs form part of the emerging so-called heterogeneous networks, hereafter 
referred to as HetNets. Traditionally network coverage and capacity has been provided by a layer of MBSs, whereas in HetNets MBSs operate alongside other, typically varying transmit power SBSs operating multi-standard radio access technologies and architectures. SBSs are primarily added to increase capacity in hotspots with high demand as well as to provide coverage fill for areas not covered by the macro cell both indoors and outdoors. This results in a heterogeneous network with MBSs and SBSs providing increased data rates per unit area. Figure.1. shows an SBS being used to provide coverage at the cell edge which may be difficult to cover from the MBS.

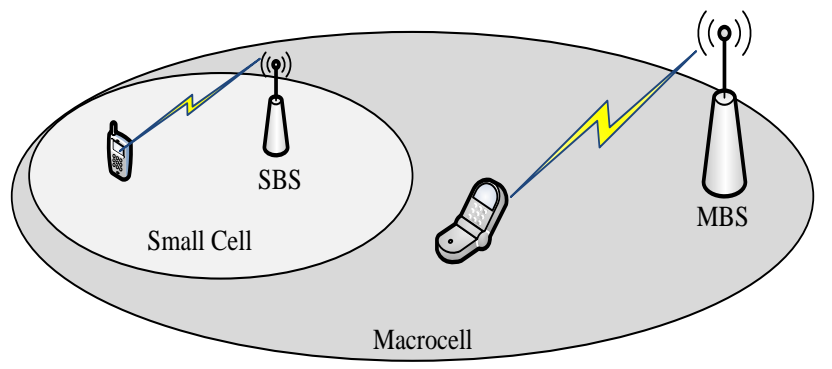

Figure 1: Heterogeneous Network

The sending of SBSs offers the administrator one of a kind choice with respect to the utilization of utilizing the equivalent or diverse recurrence groups as the encompassing or overlaying macrocells. This decision significantly affects the impedance level and a definitive execution of both the macrocell and little cell systems. While the entrance technique and recurrence use will at last influence the exhibition of the system, the choices to be made are not direct. Open access is by and large thought to be better than shut access from a limit and versatility the board perspective [43]. The decision of recurrence is affected by the RF range accessibility to a system administrator. The formation of little cell systems offers ascend to two layers, or levels, in the remote system: the macrocell layer and the little cell layer. It is very much perceived that a key test in the reception of little cell systems is the supposed cross-level obstruction between the little cell and large scale cell organize [6][45]. This is especially testing when the little cell systems share a similar recurrence band as the encompassing macrocell arrange (otherwise called general recurrence re-use) and specifically when SBS is conveyed haphazardly. This implies SBSs may cause and endure co-channel impedance with the overlaying MBSs.

Attributes of little cells, their difficulties, and potential misuses and contemplations when executing a different heterogeneous femtocell plan can be outlined as pursues:

1. SBSs may share same carrier frequencies as overlaying macro cell network giving rise to co-channel interference particular since they may be randomly deployed.

2. SBSs may be closely co-located with other SBSs sharing the same carrier frequencies giving rise to co-channel interference.

3. Users of little cells will probably be either stationary or moderate moving. For this situation, the Doppler recurrence move will be non-existent for every single pragmatic reason.
4. Given short SBS to UE removes, a high precise spread of UEs encompassing the SBS is more probable. This is not quite the same as ordinary MBSs where for the most part the base station asset is part into different segments or circular segments by fitting heterogeneous femtocell systems.

5. The dimension of an imposed for the form factor is stronger SBS heterogeneous femto cell design compared to MBS.

6. The complexity of the SBS and its Cost of heterogeneous femto cells are also more important factor compared to MBS.

7. Users are unlikely to be randomly scattered in residential small cells, they will be in one of a few fixed locations.

8. Users will be more randomly scattered in dense enterprise and public small cells.

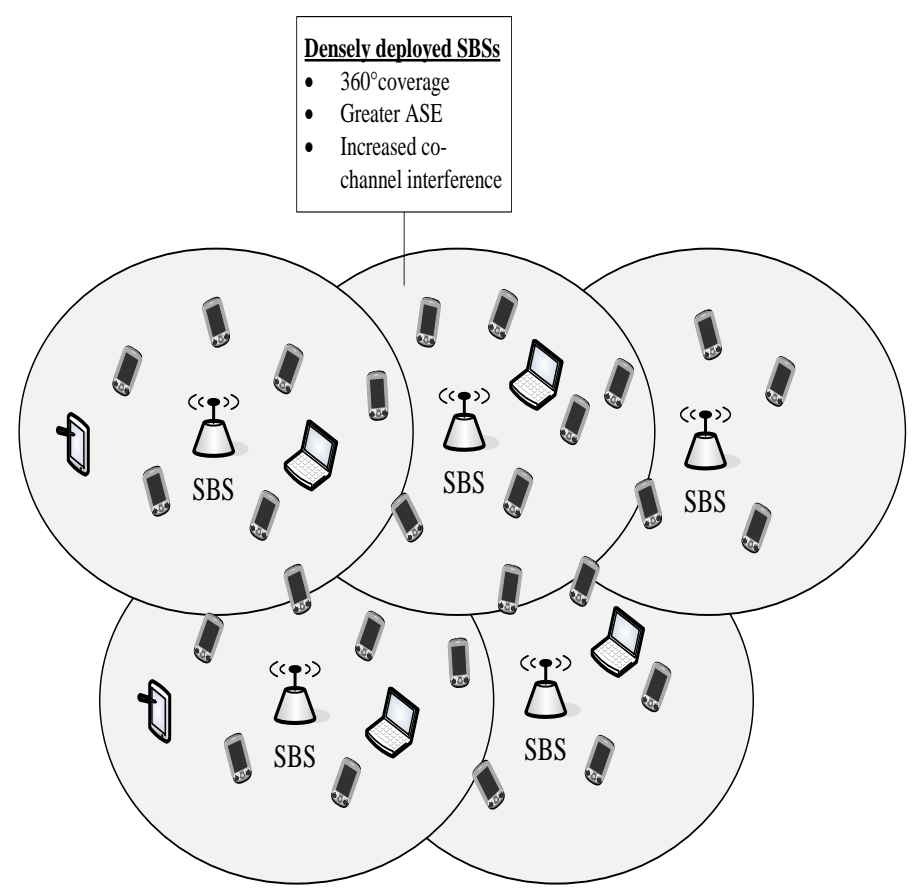

Figure 2: SBS Dense Deployment

Based on the discussion presented, this work aims to investigate the use of low complexity, energy-efficient multiple heterogeneous femtocell techniques applicable to SBSs. Emphasis is given to downlink (DL) methods i.e. the transmit direction emanating from the SBS to the UE or UEs and particular attention is given to indoor installations, where the propagation environment is expected to be uniquely challenging. The performance of the SBS to UE link is considered. More specifically, schemes that can reduce transmit power but provide the same quality of service (QoS) as a single heterogeneous femtocell system are considered, these include heterogeneous femtocell beamforming and diversity schemes. Based on the transmit power reduction, the overall SBS BS power budget and EE is determined. The work assumes that the methods to reduce the transmit power are performed, largely in the baseband portion of the SBSs. Consideration is therefore given to minimizing the operations required in this area. These include the complexity of the 
signal processing operations, the overhead in terms of channel estimation and the number of signal processing chains supporting each heterogeneous femtocell element. Furthermore, a comparative analysis is provided between one multiple heterogeneous femtocell method and another to provide an overall power usage for the same QoS. Here, the focus is given to signal processing architectures targeted specifically for the SBS. Where fitting the EE-SE exchange off, is considered as a major aspect of the investigation. The Shannon data theoretic limit with regards to point-to-point added substance Gaussian background noise has built up the presence of a basic exchange off among EE and SE considering optimistic power utilization models for example it considers just the transmit control. The use of real-world power consumption models, providing total consumed power is used in this analysis. In the investigation, the exploitation of practical small cell operating parameters is considered. These include the following:

- Low mobility

- Reduced cell radius

- Reduced SBS physical characteristics/form factor

- Transmit power level

- Heterogeneous femto cell correlation level

- Deployment densification level

- Physical layer techniques employed

Considering that the SBS will operate with much lower transmit power, the impact of the power amplifier (PA) will be much less than in the case of the MBS where, together with the feeder, it dominates the power budget e.g. 65\% [28]. This means that in the case of the SBS, the signal processing and associated transceiver processing behind each heterogeneous femtocell has more of a significant impact on the overall power budget - up to 38\% of an LTE FBS [29]. Focusing on this area and specifically targeting low complexity architectures and precoding schemes for multiple heterogeneous femtocell systems is therefore of importance and impacts the overall power budget of the SBS significantly.

\subsection{Energy Metrics}

Energy metrics are classified or metrics or energy consumption metrics as either EE or not. An EE metric is the ratio of the accomplished effort to the spent resources, whereas energy consumption metric is definite as the inverse of the EE metric i.e. a ratio of the consumed resource to the performed work. The consumed resource is the energy (Joule) or power (Watt) while the performed work could be the transferred bit rate, the number of served users, coverage area. Both metrics contain the same information but they are expressed in different ways.

\subsection{Energy Consumption Metrics}

The energy (E) per data packet bit (I), $E_{b}$, is the most common energy metric. This is given as the ratio amongst the average power consumed $(P)$ and the average data rate $(R)$. Hence $E_{b}$ given as the Joule per bit or Watt/bps.

$E_{b}=\frac{E}{I}=\frac{P}{R}=\left[\frac{\text { Joule }}{\text { bit }}\right]$ or $\left[\frac{\text { Watt }}{\text { bps }}\right]$

Similarly the energy ingesting ratio (EIR) is given per each and every packet data bit

$E C R=\frac{E}{M}=\frac{P T}{M}=\frac{P}{D}[U /$ bit $]$

where $\mathrm{E}$ is the vitality required to convey $\mathrm{M}$ bits of data after some time $\mathrm{T}$, and $\mathrm{D}=\mathrm{MT}$ is the information rate in bits every second. This furnishes us with the vitality utilization in Joules expended for transportation of one data bit. The measurement permits the supreme exhibition of various remote systems or sub frameworks to be aligned [31]. As a basic model, an ordinary LTE base station segment may work over a transfer speed of $10 \mathrm{MHz}$ with a normal ghostly proficiency of 1.5 bits $/ \mathrm{s} / \mathrm{Hz}$, in this manner accomplishing a normal information rate of $15 \mathrm{MBits} / \mathrm{s}$. On the off chance that a base station heterogeneous femto cell transmits $8 \mathrm{~W}$ of RF control, at that point the RF ECR esteem for this framework would be 0.53 microJoules per bit. Be that as it may if the absolute RF power is $150 \mathrm{~W}$ the ECR would increment to 10 microJoules per bit. Also another measurement, the vitality utilization gain (ECG) is a relative measure as opposed to a flat out one [31] and is valuable for looking at two changed frameworks. For instance one may wish to consider a SBS with a solitary transmitting heterogeneous femto cell contrasted with one with two heterogeneous femto cells. The ECG is essentially the proportion (E1/E2) where E1 is a reference framework for example single heterogeneous femto cell SBS and E2 is the two heterogeneous femto cell framework. The bigger the estimation of the ECG then the more productive framework two progresses toward becoming. To decide the decrease in vitality utilization between two unique frameworks, it is additionally helpful to consider the vitality decrease gain (ERG) which can be found as:

$E R G=\frac{E C R_{1}-E C R_{2}}{E C R_{1}} \cdot 100 \%$

where $\mathrm{ECR}_{1}$ and $\mathrm{ECR}_{2}$ correspond to the energy consumption ratio of two different systems.

\subsection{EE Metric}

The bit-per-joule capacity is an EE metric which measures the maximum amount of bits that are delivered by the network per Joule it consumed to do so and can be expressed as follows:

$C_{I}=\frac{I}{E}=\frac{R}{P}=\left[\frac{\text { bit }}{\text { Joule }}\right]$ or $\left[\frac{\text { bps }}{\text { Watt }}\right]$ 


\subsection{The SE-EE Trade-off}

SE is a generally utilized exhibition pointer for the plan of remote correspondence frameworks. SE situated frameworks are intended to expand SE under pinnacle or normal power limitations, which may prompt transmitting with the most extreme permitted control for an extensive stretch and in this manner digress from the vitality productive plan. EE is normally characterized as data bits per unit transmit vitality. For an added substance white Gaussian commotion (AWGN) channel, it is outstanding that for a given transmit power, Pt, and framework data transfer capacity $\mathrm{W}$ the channel limit is:

$R=\frac{1}{2} \log _{2}\left(1+\frac{P_{t}}{N_{0} W}\right)$

per degrees of freedom (DOF), where $N_{0}$ is the noise power spectral density. According to the Nyquist sampling theory, $\mathrm{DOF}$ is $2 \mathrm{~W}$. Consequently, the channel capacity is $C=2 \mathrm{WR}$ bits per second. The channel capacity in bits/s is given by:

$C=W \log _{2}\left(1+\frac{P_{t}}{N_{0} W}\right)$

Consequently $\mathrm{SE}$ is given by:

$\eta_{S E}=\log _{2}\left(1+\frac{P_{t}}{N_{0} W}\right)$

Consequently EE is:

$\eta_{E E}=W \log _{2}\left(1+\frac{P_{t}}{N_{0} W}\right) /\left(P_{t}\right)$

As a result, the SE-EE relation can be expressed as:

$\eta_{E E}=\frac{\eta_{S E}}{\left(2^{\eta S E}-1\right) N_{0}}$

$\eta_{E E}=\frac{C}{P_{t}}=\frac{2 R}{N_{0}\left(2^{2 R}-1\right)}$

From (10), it can be shown that $\eta_{E E}$ decreases monotonically with $\mathrm{R}$. With $\max \eta_{E E}=1 /\left(N_{0} \ln 2\right)$ as $\mathrm{R} \rightarrow 0$, and $\min \eta_{E E}=0$ as $\mathrm{R} \rightarrow \infty$ Error! Reference source not found.

The results obtained from

(10) accept a limitless size of data square and boundless number of DOF. In the limited case the conduct will be unique. By and by the EE limits from the data theoretic examination probably won't be accomplished in useful frameworks because of execution loss of limit moving toward channel codes, for example, Turbo codes, defective information of the CSI, the exhibition of the synchronization subsystem and transmission related electronic circuit utilization. When considering the circuit control EE should be re-characterized as data bits per unit vitality for example not simply the transmitted vitality, where extra circuit control, Pc, should be incorporated:

$$
\eta_{E E}=\frac{2 R}{P_{c}+N_{0}\left(2^{2 R}-1\right)}
$$

The EE-SE trade-off is shown in Figure.3. In particular, circuit power will break the monotonic relation between SE and EE.
If circuit power is considered it will turn the curve into a bell shape.

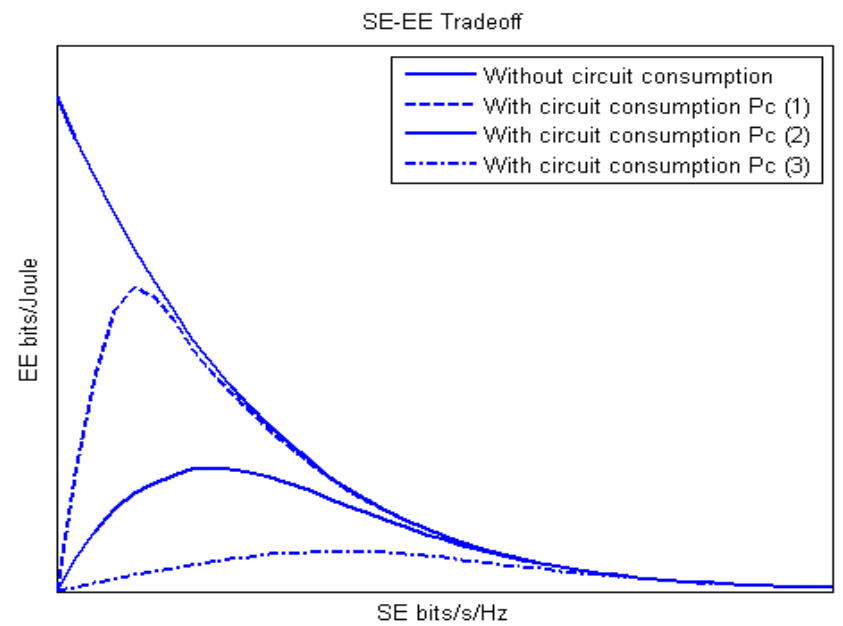

Figure 3: EE/SE trade-off in AWGN

The investigation can be stretched out to consider the intensifier effectiveness $\rho$ of the transmitting sub-framework. Since EE is the channel limit standardized by the framework control utilization, where the absolute power utilization is a whole of $\mathrm{Pt}$ and the circuit control $\mathrm{Pc}$ it tends to be communicated as a component of intensifier proficiency [39].

$\eta_{E E}=\frac{W \log _{2}\left(1+P_{t} /\left(N_{0} W\right)\right)}{P_{t} / \rho+P_{c}}$

where $\rho$ is the power amplifier efficiency. Although SE is the channel capacity normalized by the system bandwidth, the relationship of EE and SE can be shown as:

$\eta_{E E}=\frac{W_{\eta S E}}{P_{c}+N_{0} W\left(2^{\eta S E}-1\right) / \rho}$

The association of EE and SE as a function of power amplifier efficiency is shown in Figure.4. The illustration, plotted logarithmically, again shows the expected monotonic behavior between $\mathrm{SE}$ and $\mathrm{EE}$ when $\mathrm{P}_{\mathrm{c}}$ is zero. With $\mathrm{Pc}$ non-zero, EE increments in the low SE district and diminishes in the high SE locale which implies that for a given EE there exists two SE esteems [39]. Because of the circuit control and without a doubt the PA, the perspective on EE changes the perspective on executions, for example, MIMO where there will be an expansion and intricacy prompting expanded power utilization. In a perfect world, the EE-SE bend in future frameworks ought to endeavor to meet the accompanying necessities [39]:

$>$ EE-worth ought to be improved for every SE activity point.

$>$ EE-SE win-win district ought to be amplified and the EE-SE exchange off area ought to be diminished.

The slant of the EE-SE exchange off area ought to be diminished. 


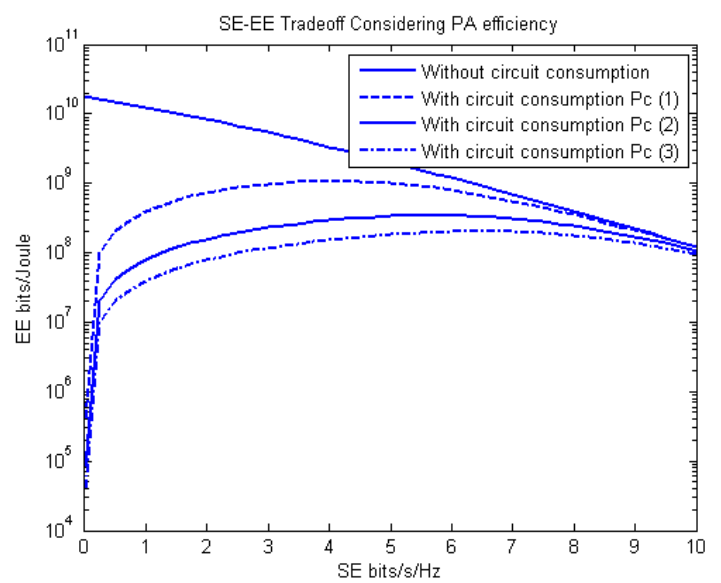

(a)

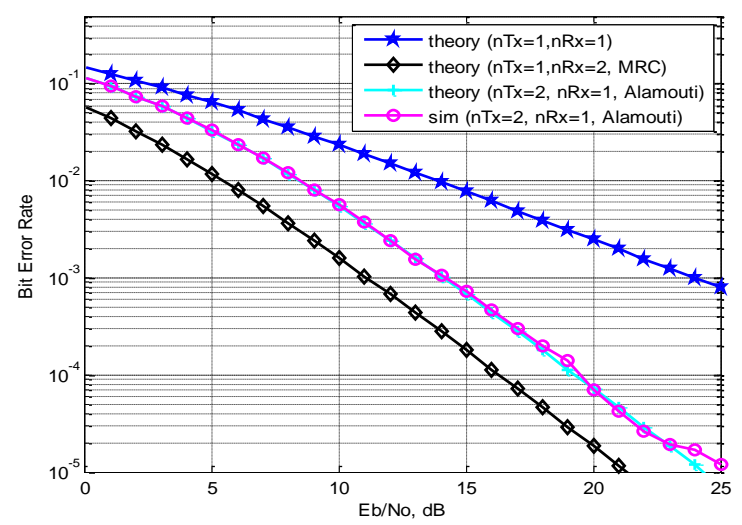

(b)

Figure 4: (a) EE/SE Trade-off as a function of Amplifier Efficiency Figure.5 (b)BER of proposed H-RTP and H-RTCP protocol and its Performance in Rayleigh Fading

Since the Network Simulator (NS2) model was independently developed as part of this research, validation of the model was firstly required. Without the complication of frequency selectivity, the model's validity was provided by considering less complex channel types. Consideration of AWGN only and then a flat faded Rayleigh channel were first used to assess the model performance. It is noted that RTP and RTCP stated that the performance curves can be used to predict performance regardless of the employed coding and modulation schemes used. This tells us that the simulation of the W-CDMA model should, therefore, provide an equivalent performance improvement as Figure. 5. Assuming a single tap, flat Rayleigh channel is used. The simulation results of this are shown in Figure.9. When comparing the $1 \%$ error rate point, there is an approximate $5 \mathrm{~dB}$ of gain using RTP and RTCP compared to SISO. This, therefore, aligns very well with the theoretical results of Figure 3.1 and the claim by RTP and RTCP. The performance of RTP and RTCP is critically dependent on the availability of independent multiple channels. Channel correlation will downgrade the performance of an RTP and RTCP system, especially it's capacity. Channel correlation is a measure of the similarity or likeness between the channels of the between transmit and received heterogeneous femtocell of the system. In the extreme case, where the channels are fully correlated, the RTP and RTCP system will perform no differently from a single heterogeneous femtocell configuration. The capacity of an RTP and RTCP system not only depends on the number of channels (NxM) but also depends on the correlation between the channels. In general, the greater the channel correlation, the smaller the channel capacity. The channel correlation of an RTP and RTCP system is mainly due to two components: Spatial Correlation, Heterogeneous femtocell Mutual Coupling. Only the spatial correlation is considered in the following analysis.

The IoT structures may contain inactive likewise, dynamic centers. This suggests the intelligent media data might be transmitted through imperativeness based center points or common centers. Concerning standard center points, the essentialness isn't a fundamental factor. For the imperativeness based centers, IoT-RTP must know about the conditions of these sorts of centers. As such, a field must be incorporated in the IoT-RTP to record the imperativeness level for every center point in the intelligent media session.

\section{Proposed algorithm: Combined IoT-RTP and RTCP-IoT}

$\mathrm{U}$ is the number nodes(users) used as multimedia metadata in the session

$\mathrm{N}$ :No of sessions in sub-module

I: No of Interior sub sessions

$\mathrm{S}$ : Capacity in above sessions

$\mathrm{S}_{\text {div }}$ : Network Capacity division of I

$\mathrm{N}_{\mathrm{f}}$ : No.of flows in I

if $\sum_{i=1}^{U}$ sessionid $\leq S$

Wait initial stage itself

or

for $\mathrm{X}=1$ to $\mathrm{N}$

begin

$$
\begin{gathered}
\text { if } \left.\sum_{j=1}^{U d P} \text { session } I d \leq C_{d i v}\right) \\
\text { Wait initial stage itself }
\end{gathered}
$$

$$
p f_{i}=\frac{\text { session } i d}{F}
$$

Wait initial stage itself

or

for $\mathrm{i}=1$ to 4

$p f_{i}$ Need to be transmitted through RTP and store in the cloud Though (thingspeak.com)

end

End of the algorithm

\section{IoT RTCP}

The versatile assortment of RTCP in like manner contemplates the state of the IoT structure concerning the transmission of blended media streams. In like manner, its bits of knowledge 
Mohan Babu.C et al., International Journal of Advanced Trends in Computer Science and Engineering, 9(2), March - April 2020, 2065 - 2077

assemble information around resources that may start in the IoT structures and that move from various plans. It may change other than considers the kind of center centers (dynamic or latent). The specs of everything in the IoT structure, for instance, overseeing, memory, and criticalness, should be thought of. Likewise, unprecedented blended media coding must be reflected. Minimization of RTCP bits of knowledge without influencing the sight and sound correspondence complete IoT structures is a fundamental obsession in RTCP, especially if there ought to be an event of framework famishment. To accomplish the destinations, the prioritization of control reports should be connected. In the standard RTCP, there are two essential sorts of reports, unequivocally sender report (SR) and beneficiary report (RR). SR contains various segments, for instance, the proportion of transmitted packages inside a period, the various fields of the RTCP, for instance, Network Time Protocol (NTP), and synchronization sources, timestamps. In RR field contains various fields, for instance, separate lost (FL), studied number of social affairs expected (NPE), and between landing jitter. In SR, RR and RTCP must be restored by social affair various fields to accumulate express data around the IoT structure. In like manner, these bits of knowledge will be bestowed under the kept condition to tie the over-disturbing of the additional fields. As passed on above in the genuine idea of IoT-RTP, IoT-RTCP other than limits the adroit media meeting into a get-together of clear meetings. All meetings have an executive that is picked using the framework uncovered. The division of blended media meeting procedures should be constrained with meeting measure [1]. In the event that the sight and sound meeting measure is more noticeable than an appointed edge, For any circumstance, by reasonability of a standard sight and sound meeting, the division system will be ignored. The edge what's dynamical, conventional sizes are depicted in the reenactment area.

\section{Proposed algorithm: RTCP-IoT with Cloud storage}

AT: Allocated time for getting of the bundle in the interim on the gathering of the report (RR).

UL: Upper degree of complete mixed media divisions. ML:After Separation of the Middle level meetings.

NL: After Separation of the Lower level of meeting. EED:end-end-delay.

PL:packet misfortune.

J:Jitter with delay

$\mathrm{R}:$ Report on the gathering of the bundle

start

for $\mathrm{K}=1$ to $\mathrm{UL}$

start

$$
\begin{aligned}
& \text { for } \mathrm{Z}=1 \text { to } \mathrm{M} \\
& \text { start } \\
& \text { for } \mathrm{Q}=1 \text { to } \mathrm{N}
\end{aligned}
$$

start

if(EED, PL, J are standard rationales)

start

IoT-RTCP stoped the extra fields

$$
\begin{aligned}
& \text { Report on the gathering of the bundle } \\
& R_{K}=R_{Z}+R_{Q} \\
& \text { end } \\
& \text { else } \\
& \text { begin }
\end{aligned}
$$

AT updated to increase and additions are included for the RTCP and send $R_{K}=R_{Z}+R_{Q}$

$$
\text { end }
$$$$
R_{M}=R_{U L}+R_{Z}
$$

end

$R_{U L}=R_{Z}+R_{M}$ then receive the packets from the cloud and plots in the NS2 for validations of multimedia datasets

end for termination

\section{ALGORITHM/ PROPOSED METHODOLOGY}

The proposed strategy/calculation can be extensively ordered into following:

Stage 1: select the patient for examination

Stage 2: read the information from the different sensors embedded to quiet.

Stage 3: By accepting the information from various sensors by making remote body territory arrange.

Step4: begin transmitting information utilizing RTP/RTCP.

Stage 5: examinations the information for transferring to cloud Stage 6: get information through distributed storage utilizing same convention during transmission.

Stage 7: RTP-RTCP framework for helping the information offer to master.

Step8: remote access by the specialist

Step9: create the individual solution for a specific patient

Stage 10: Repeat step4 to stage 6

Stage 11: the comparing solution will reach through the sheltered and effective channel to the Patient overseer.

\section{SIMULATION RESULTS}

Simulation results in the form of SINR cumulative distribution function (CDF) based on, bit error rate (BER) measurements and capacity/rate calculations based on were taken at the UE associated with the SOI. These provided the necessary inputs to determine the power reduction possible when compared to a single heterogeneous femto cell. SINR CDF results in Error! Reference source not found.6 - Error! Reference source not found. show the performance relative to the cell spacing at the SOI UE for the 50\% loaded case. Each graph shows a CDF curve for each heterogeneous femto cell spacing/CSI method combination. Since the performance between each case is very close or overlaps only the best and worst cases have been annotated. The performance relative to the single directional heterogeneous femto cell case is shown. The impacts on SINR are evident for each heterogeneous femto cell spacing and the spread in SINR from the worst case to the best performer is shown to range from $3.5 \mathrm{~dB}$ for the two heterogeneous femto cell to $11 \mathrm{~dB}$ for the four heterogeneous femto cell case. We note that for the two heterogeneous femto cell, $f_{d}=0.01 \mathrm{~Hz}$ case, the all-directional heterogeneous 
femto cell performance is better (has a higher SINR) than all beam formed cases using single heterogeneous femto cell CSI. For the two heterogeneous femto cell, $f_{d}=1.95 \mathrm{~Hz}$, the increase in Doppler frequency shows all beam forming cases outperform the all-directional case. For the four heterogeneous femto cell case all-directional, performance is close to single heterogeneous femto cell beam forming with element spacing of $0.25 \lambda$. As in the two heterogeneous femto cell, four heterogeneous femto cell beam forming performance improves with increased Doppler

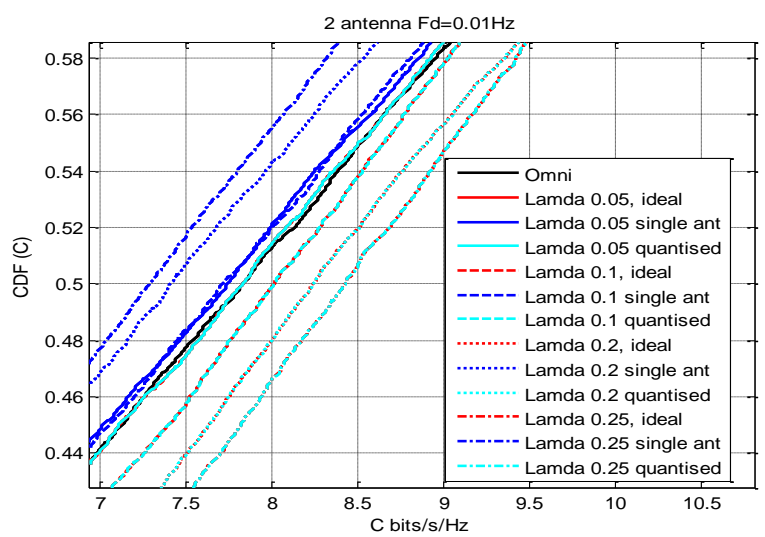

Figure.6: Two heterogeneous femto cell SINR CDF

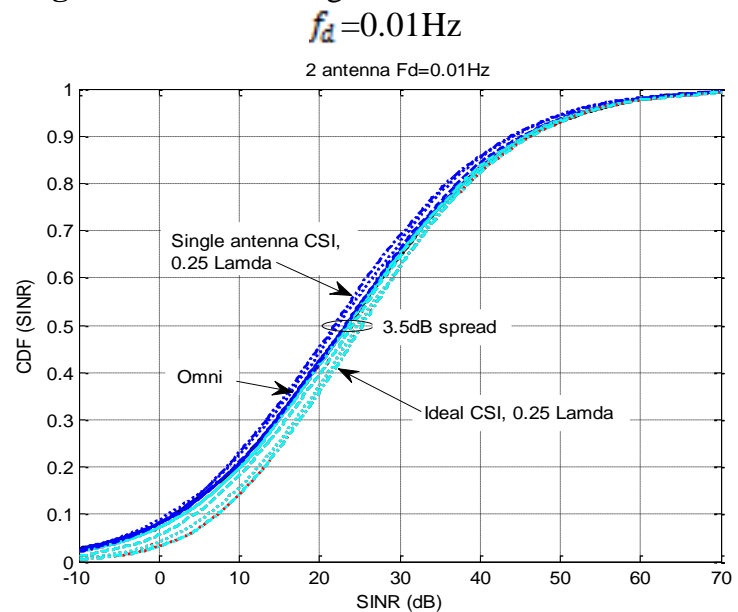

Figure 7:Two heterogeneous femto cell

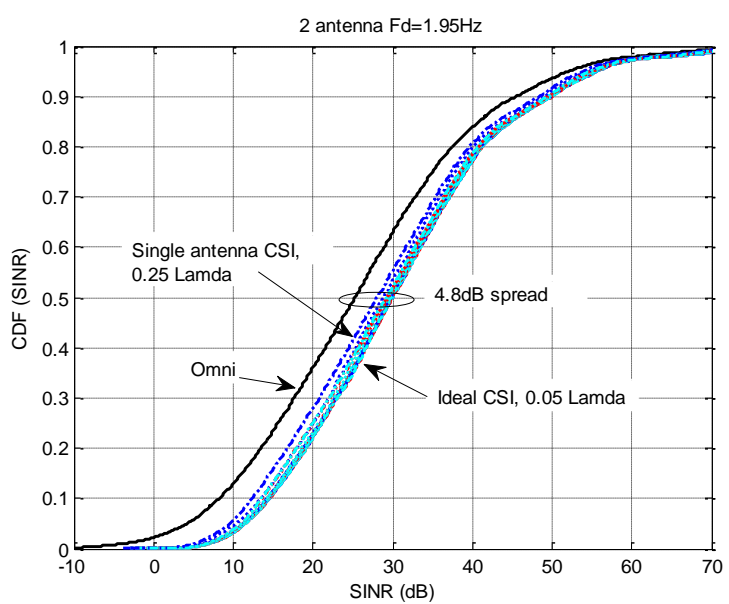

Figure 8:.Two heterogeneous femto cell SINR CDF

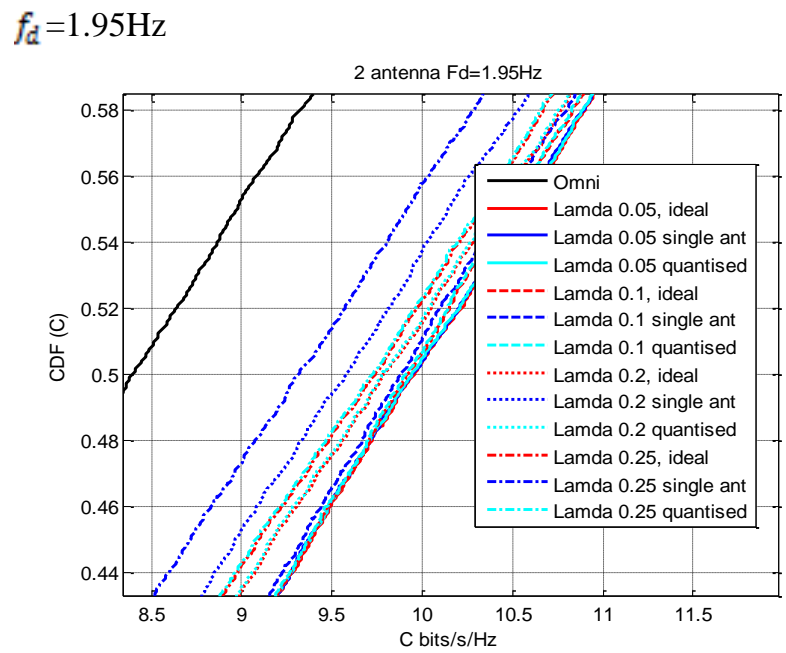

Figure 9: Two heterogeneous femto cell

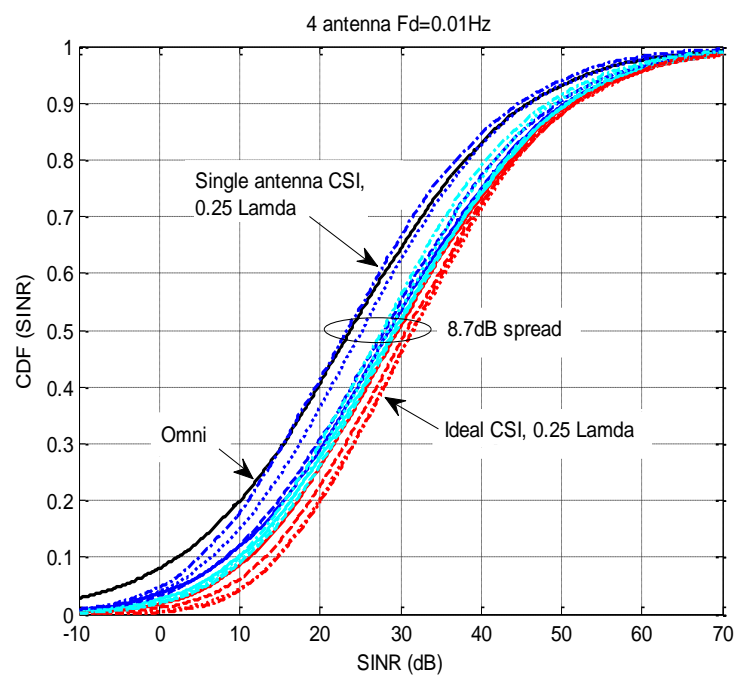

Figure 10:.Four heterogeneous femto cell SINR CDF $f_{\mathbb{Q}}=0.01 \mathrm{~Hz}$

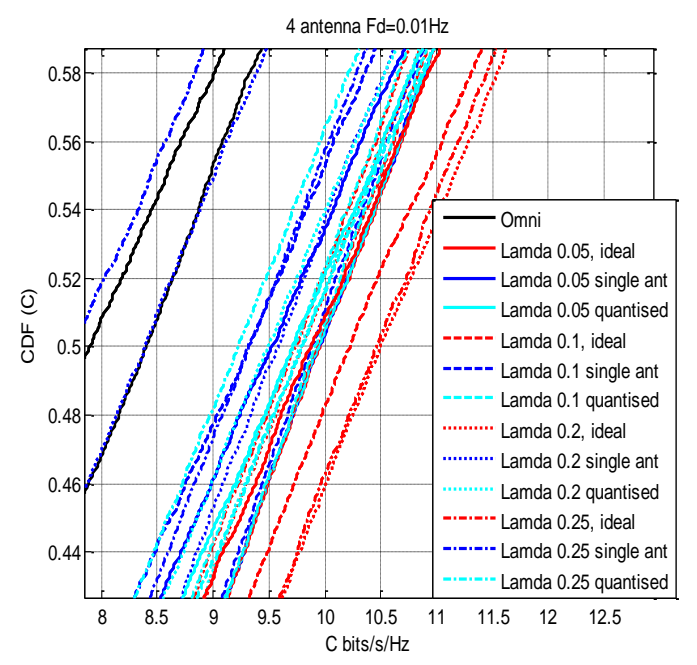

Figure 11:.Four heterogeneous femto cell 


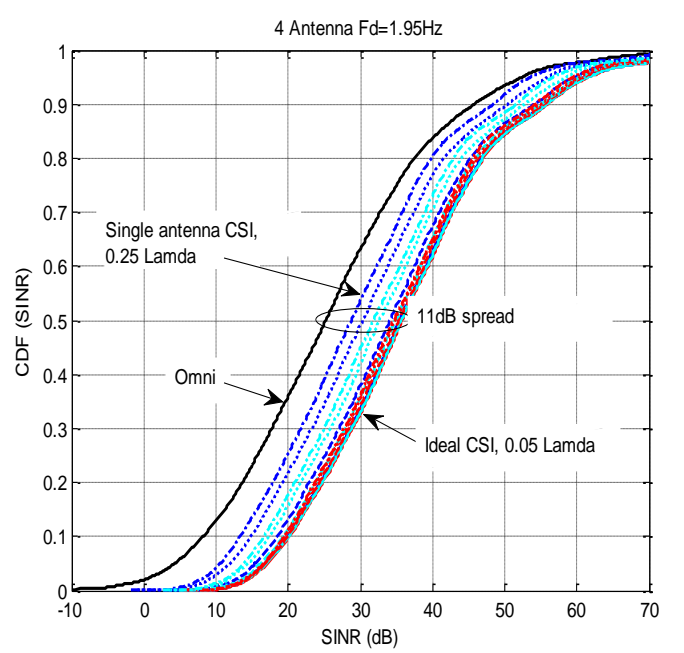

Figure 12.:Four heterogeneous femto cell SINR CDF,

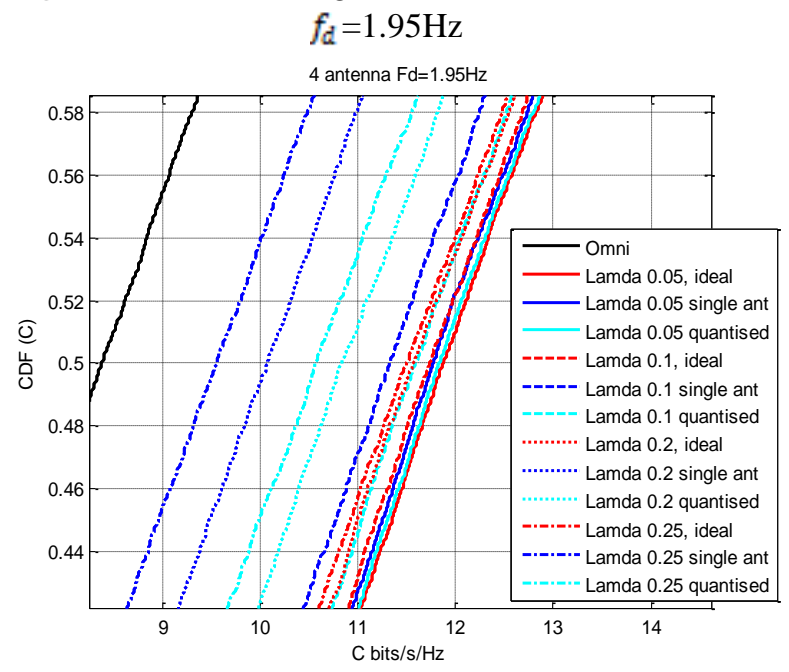

Figure 13:.Four heterogeneous femto cell

\section{CONCLUSION}

This research work presents another heterogeneous system using RTP-RTCP in which LTE and Wi-Fi remote gadgets are used so as to profit by the data transfer capacity of every transmission innovation. What's more, another directing convention for heterogeneous remote work systems is created, which chooses powerfully the transmission innovation so as to expand the general system limit and upgrade the normal throughput. Also, another steering calculation is proposed for the requirements of the directing convention, which gauges the expense of transmitting the traffic through each system. The proposed computation considers the traffic load on the LTE orchestrates as an estimation to assess the cost of transmission over LTE and usages transmission rate as an estimation for the Wi-Fi work arrange. The reenactment results show that the proposed framework achieves up to $150 \%$ more throughput differentiated and Wi-Fi-just frameworks and LTE-just frameworks. The heterogeneous framework configuration manages assorted remote contraptions as a bit of a solitary virtual framework. The LTE framework is utilized to avoid stopped up Wi-Fi center points and high impediment route in the WMN while the WMN offloads the load of the LTE sort out, diminishes the cost of using more grant repeat gatherings and advances the data to another center when the LTE throughput is defiling. This work gives the foundation of future research on making heterogeneous Wi-Fi/LTE work frameworks and using diverse remote advancements as an element of heterogeneous frameworks. The proposed directing convention can possibly be reached out to help different remote advancements by using their parameters in the learning calculation. The proposed design gives a simple method to grow the versatile system inclusion and limit and could add to the 5G framework. In addition, the heterogeneous systems could be utilized to interface the Internet of Thing systems and utilized to give the framework to savvy homes and keen urban areas.

\section{REFERENCES}

[1]. IWPC International Wireless Industry Consortium, "Developmental and troublesome dreams towards ultra-high limit systems," pp. 1-89, 2014.

[2]. I.F. Akyildiz, X. Wang, and W. Wang, "Remote work arranges: a study," Journal of Computer Networks, pp. 445-487, vol. 47, issue 10, 2005. https://doi.org/10.1016/j.comnet.2004.12.001

[3]. N. Himayat, S. Yeh, and A. Panah, "Multi-radio heterogeneous systems: models and execution," In International Conference on Computing, Networking and Communications (ICNC), pp. 252-258, Honolulu, HI, USA, February, 2014.

[4]. Ahmed Al-Saadi.et.al, "Steering Protocol for Heterogeneous Wireless Mesh Networks", IEEE Transactions on Vehicular Technology, PP-0018-9545, IEEE, 2015.

[5]. Z. Yang, Q. Yang, and F. Fu, "An epic weight altering plan in LTE moreover, WiFi existed together framework for OFDMA system," In Proceedings of Global Conference on Wireless Communications and Signal Processing, pp. 1-55, Hangzhou, China, 2013.

[6]. D.H. Hagos, and R. Kapitza, "Focus on execution driven offload strategies for LTE frameworks," In Proceedings of IEEE 6th Joint IFIP Wireless and Mobile Networking Conference (WMNC), pp. 1-10, Dubai, UAE, 2013.

[7]. L. Hu, C. Colett, N. Huan, I.Z. Kovács, B. Vejlgaard, R. Irmer, and N. Scully, "Reasonable indoor Wi-Fi and Femto course of action consider as the offloading answer for LTE enormous scale frameworks," In IEEE Vehicular Technology Conference (VTC Fall), pp. 1-6, Quebec City, QC, Canada, 2012.

[8]. 3GPP, Group Service and System Aspects Service Requirements for Evolution of 3 GPP System (Rel.8), 3GPP TS 22.278, Dec. 2008.

[9]. WiFi (Wireless Fidelity), IEEE 802.11 WG, IEEE 802.11-2007, Wireless LAN MAC and PHY assurance, revision of IEEE 802.11 1999, IEEE LAN/MAN Standards Committee, June, 2007. 
[10].D.B. Johnson, D.A. Maltz, and Y.- C. Hu, "The dynamic source coordinating show for adaptable uncommonly named frameworks (DSR)," The IETF Trust, February, 2007.

[11].C. E. Perkins and P. Bhagwat, "Incredibly remarkable objective sequenced evacuate vector guiding (DSDV) for adaptable PCs," In Proceedings of SIGCOMM Conference on Communications Architectures, Protocols and Applications, pp. 234-244, New York, USA, August, 1994.

[12].T. Clausen and P. Jacquet, "RFC 3626: Optimized association state controlling show," October, 2003.

[13]. M Abolhasan , T Wysocki, E Dutkiewicz, "A study of controlling shows for adaptable exceptionally named frameworks," Ad Hoc Networks ,pp. 1-22, vol 2, issue 1, 2004.

https://doi.org/10.1016/S1570-8705(03)00043-X

[14].H. Wu, C. Qiao, S. De, and O. Tonguz, "Joined cell and unrehearsed giving off systems: iCAR," IEEE Journal on Selected Areas in ommunications, vol. 19, issue 10, pp. 2105-2115, October, 2001

[15].S. Dixit, E. Yanmaz, and O.K. Tonguz, "On the arrangement of self-made cell remote frameworks," IEEE Communications Magazine, vol. 43, issue 7, pp. 86-93, July, 2005. https://doi.org/10.1109/MCOM.2005.1470827

[16].H. Luo, R. Ramjee, P. Sinha, L.E. Li, and S. Lu, "UCAN: a bound together cell and uncommonly selected framework designing," In Proceedings of ACM MOBICOM, pp. 353-367, San Diego, CA, USA, September, 2003.

[17].H. Li, M. Lott, M. Weckerle, W. Zirwas, and E. Schulz, "Multihop correspondences in future adaptable radio frameworks," In Proceedings of IEEE PIMRC'02, Lisbon, pp. 54-58, Portugal, September, 2002.

[18].G. Chen, M. Tune, Y. Zhang, and J. Tune, "Cross-layer change with created reserving for heterogeneous remote frameworks," IEEE 72nd Vehicular Technology Conference, pp. 1-5, Ottawa, ON, Canada, September, 2010. https://doi.org/10.1109/VETECF.2010.5594560

[19].N. Himayat, S. Yeh, and A. Panah, "Multi-radio heterogeneous frameworks: structures and execution," In International Conference on Computing, Networking and Communications (ICNC), pp. 252-258, Honolulu, HI, USA, February, 2014.

[20].L. Chou, S. Cheng, C. Li, and S. Chen, "Remote framework the administrators structures for WiMAX/Wi-Fi work frameworks," Emerging Directions in Embedded and Ubiquitous Computing Lecture Notes in Computer Science, pp. 215-225, vol. 4809, 2007.

[21]. W. Sun, P. Zhang, Y. Chen, Z. Qin, and D. Teng, "A tight coupling support plot in WiFi/WiMAX heterogeneous work frameworks," International Journal of Computers, Communications and Control, pp. 460-468, vol. 8, issue 3, 2013.

https://doi.org/10.15837/ijccc.2013.3.10
[22]. W. Wang, X. Liu, J. Vicente, and P. Mohapatra, "Joining expansion of heterogeneous WiFi/WiMAX frameworks," IEEE Transactions on Mobile Computing, pp. 1131-1143, vol. 10, issue 8, 2011.

[23].G. Zhioua, N. Tabbane, H. Labiod, and S. Tabbane, "A Fuzzy Multi-Metric QoS-Balancing Gateway Selection Algorithm in a Clustered VANET to LTE Advanced Hybrid Cellular Network," IEEE Transactions on Vehicular Technology, vol. 64, no. 2, February 2015. https://doi.org/10.1109/TVT.2014.2323693

[24].Y.S. Soh, T.Q.S. Quek, M. Kountouris, and H. Shin, "Vitality proficient heterogeneous cell systems," IEEE J. Sel. Regions Commun., vol. 31, issue 5, pp. 840-850, May, 2013.

[25].J. Lin and K. Feng, "Femtocell get to procedures in heterogeneous systems utilizing a game hypothetical structure," IEEE Transactions on Wireless Communications, vol. 13, issue 3, pp. 1208-1221, March 2014.

[26]. Tarik Taleb.et.al, "A Cross-Layer Approach for an Efficient Delivery of TCP/RTP-Based Multimedia Applications in Heterogeneous Wireless Networks", IEEE TRANSACTIONS ON VEHICULAR TECHNOLOGY, VOL. 57, NO. 6, NOVEMBER 2008.

[27]. Omar Said.et.al, "IoT-RTP and IoT-RTCP: Adaptive Protocols for Multimedia Transmission over Internet of Things Environments", 2169-3536, IEEE. Interpretations and substance mining, 2017.

[28]. Shiwen Mao.et.al, "MRTP: A Multiflow Real-Time Transport Protocol for Ad Hoc Networks", IEEE TRANSACTIONS ON MULTIMEDIA, VOL. 8, NO. 2, APRIL 2006 https://doi.org/10.1109/TMM.2005.864347

[29]. Alouini, M.- S.; Goldsmith, A.J., "Zone unearthly proficiency of cell portable radio frameworks," Vehicular Technology, IEEE Transactions on , vol.48, no.4, pp.1047,1066, Jul 1999.

[30]. Chandrasekhar, V.; Kountouris, M.; Andrews, J.G., "Inclusion in multi-radio wire two-level systems," Wireless Communications, IEEE Transactions on , vol.8, no.10, pp.5314,5327, October 2009.

[31].Andrews, J.G.; Buzzi, S.; Wan Choi; Hanly, S.V.; Lozano, A.; Soong, A.C.K.; Zhang, J.C., "What Will 5G Be?," Selected Areas in Communications, IEEE Journal on, vol.32, no.6, pp.1065,1082, June 2014.

[32]. Imran, A.; Zoha, An., Abu-Dayya, Adnan "Difficulties in 5G: how to engage SON with huge information for empowering 5G," IEEE Network, vol.28, no.6, pp.27,33, Nov.- Dec. 2014doi: 10.1109/MNET.2014

[33]. International Telecommunications Union, "ITU and Climate Change", 2008.

[34]. Ofcom, "A carbon review and environmental impression of Ofcom," Nov., 2007.

[35]. Small Cell Forum, "Little cell yearly report and audit. October 2013 to September 2014," Available: http://www.smallcellforum.org. 
[36].D. P. Malladi, "Heterogeneous Networks in 3G and 4G," IEEE Communn. Hypothesis Wksp., http://www.ieeectw.org/program.html, May 2012.

[37]. F. Cao, Z. Fan, "The tradeoff between vitality proficiency and framework execution of little cell sending," Proc. Global Symposium on Wireless Communications Systems (ISWCS), pp. 315-319, 2010.

[38].J. Soler Garrido, W H Chin, "Execution of a LTE femtocell base station utilizing uplink radio wire Selection," Wireless Advanced WiAd, 2011, pp. 224-229.

[39].F. Heliot, M. Imran and R. Tafazolli, "On the vitality productivity addition of MIMO correspondence under different power utilization models," Proc. Future Mobile and Network Summit (FutureNetw),. 2011, pp. 1-9.

[40].L. M Correia, et. al, "Difficulties and empowering advances for vitality mindful versatile radio systems," IEEE Communications Magazine, vol.48, no.11, pp.66-72, November 2010

[41].Heliot, F.; Imran, M.A.; Tafazolli, R., "On the vitality proficiency increase of MIMO correspondence under different power utilization models," in Future Network and Mobile Summit (FutureNetw), 2011 , vol., no., pp.1-9, 15-17 June 2011.

[42].J. G. Andrews, H. Claussen, M. Dohler, S. Rangan and M.C.Reed, "Femtocells: past present and future," IEEE Journal on Selected Areas In Communications, Vol. 30, No. 3, April 2012 pp. 497-508.

[43]. S.F. Hasan, N.H. Siddique, S. Chakraborty, "Femtocell versus $\mathrm{Wi}-\mathrm{Fi}$ - an overview and correlation of engineering and execution," Proc. first Internationl Conference on Wireless Communications, Vehicular Technology, Information Theory promotion Aerospace and Electronic Systems Technology, Wireless Vitae, 2009, pp. 916-920.

[44].G. de la Roche, A. Valcarce, D. Lopez Perez and J. Zhang, "Access control systems for femtocells," IEEE Communications Magazine, Vol. 48, no.1, July 2009, pp. 32-39.

[45].Zhenning Shi; Reed, M.C.; Ming Zhao; He Wang, "Uplink impedance situations in two-level systems," in Signals, Systems and Computers (ASILOMAR), 2010 Conference Record of the Forty Fourth Asilomar Conference on , vol., no., pp.471-476, 7-10 Nov. 2010. 